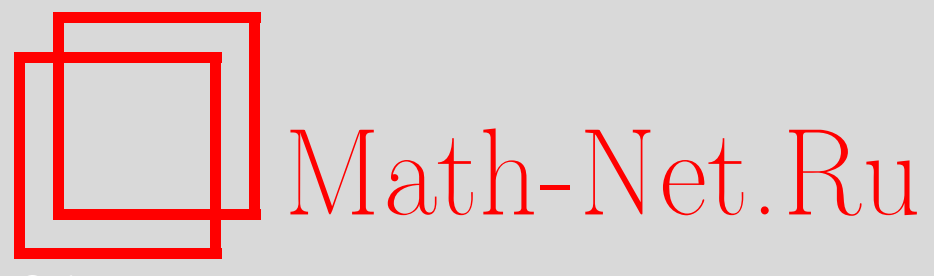

А. П. Чугайнова, Асимптотическое поведение нелинейных волн в упругих средах с дисперсией и диссипацией, TMФ, 2006, том 147, номер 2, 240-256

DOI: https://doi.org/10.4213/tmf1961

Использование Общероссийского математического портала Math-Net.Ru подразумевает, что вы прочитали и согласны с пользовательским соглашением http: //www. mathnet.ru/rus/agreement

Параметры загрузки:

IP : 18.207 .199 .55

26 апреля 2023 г., 03:42:17

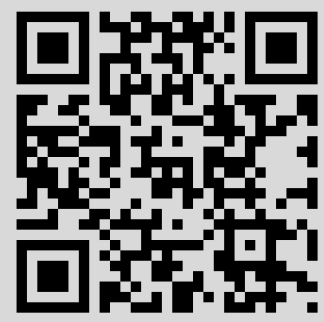




\title{
АСИМПТОТИЧЕСКОЕ ПОВЕДЕНИЕ НЕЛИНЕЙНЫХ ВОЛН В УПРУГИХ СРЕДАХ С ДИСПЕРСИЕЙ И ДИССИПАЦИЕЙ
}

\begin{abstract}
Для нелинейных упругих квазипоперечных волн в композитных средах, описываемых нелинейными гиперболическими уравнениями, изучается проблема неединственности решений такой стандартной автомодельной задачи, как задача о распаде произвольного разрыва. Система уравнений дополнена членами, описывающими диссипацию и дисперсию, влияние которых проявляется в мелкомасштабных процессах. Численно построены решения и найдены автомодельные асимптотики при больших временах полученных решений уравнений с начальными данными в виде "размазанного" разрыва. Выявлены закономерности реализации той или иной автомодельной асимптотики в зависимости от выбора начальных условий, в том числе, от вида функций, задающих мелкомасштабное сглаживание начального разрыва.
\end{abstract}

Ключевые слова: нелинейные гиперболические уравнения, ударные волны, диссипация, дисперсия.

Рассмотрим особенности поведения нелинейных квазипоперечных волн в упругих композитных средах, в которых длинноволновые возмущения описываются нелинейными гиперболическими уравнениями, выражающими законы сохранения. В решениях этих уравнений в процессе их эволюции возникают разрывы. В явлениях более мелкого масштаба существенны дисперсия и диссипация. Для описания процессов, происходящих в узких зонах, которые в большом масштабе заменяются разрывами, будем использовать более полную систему уравнений, учитывающую крупномасштабные и мелкомасштабные явления. Разрывы, которым соответствует непрерывное решение типа бегущей волны полной системы уравнений (решение задачи о структуре разрыва), обычно называются допустимыми. Влияние дисперсии и диссипации приводит к тому, что множество допустимых разрывов приобретает сложное строение. Пусть в качестве модели крупномасштабных явлений принята гиперболическая система уравнений и связанная с ней система соотношений на разрывах с дополнительным требованием того, что разрывы принадлежат множеству допустимых разрывов. Тогда, как показано в работах [1]- [3] для нелинейных

* Математический институт им. В. А. Стеклова РАН, Москва, Россия. E-mail: A.P.Chugainova@mi.ras.ru 
волн в стержнях и в магнетиках, а также для некоторой модели композитной среды, решения автомодельных задач для выделенных областей исходных параметров оказываются не единственными. Число решений в этих областях зависит от дисперсии внутри структуры разрывов (при постоянной вязкости) и неограниченно растет с ростом дисперсии. В данной работе проведен численный анализ автомодельных асимптотик в областях неединственности как результата эволюции нестационарных решений полной системы уравнений в частных производных.

\section{1. МОДЕЛЬ ЯВЛЕНИЙ КРУПНОГО МАСШТАБА}

Слабонелинейные квазипоперечные волны, распространяющиеся в положительном направлении оси $x$, при малой анизотропии среды могут описываться упрощенной системой уравнений, которая следует из системы уравнений нелинейной теории упругости [4], [5]:

$$
\frac{\partial u_{\alpha}}{\partial t}+\frac{\partial}{\partial x}\left(\frac{\partial R\left(u_{1}, u_{2}\right)}{\partial u_{\alpha}}\right)=0
$$

где

$$
\begin{gathered}
u_{\alpha}=\frac{\partial w_{\alpha}}{\partial x}=u_{\alpha}(x, t), \quad \alpha=1,2 \\
R\left(u_{1}, u_{2}\right)=\frac{1}{2} f\left(u_{1}^{2}+u_{2}^{2}\right)+\frac{1}{2} g\left(u_{2}^{2}-u_{1}^{2}\right)-\frac{1}{4} \kappa\left(u_{1}^{2}+u_{2}^{2}\right)^{2},
\end{gathered}
$$

$w_{\alpha}$ - перемещения частиц, рассматриваемые как функции лагранжевых координат $x_{1}, x_{2}, x_{3}=x$; константы $g, \kappa, f$ имеют следующий смысл: $g>0$ - параметр анизотропии, $\kappa$ - постоянная, имеющая размерность скорости, которая характеризует нелинейные эффекты, $f$ - характеристическая скорость при отсутствии нелинейности и анизотропии (при $\kappa=0, g=0)$. Знак упругой константы $\kappa$ существенно влияет на поведение квазипоперечных простых и ударных волн. В данной работе предполагается, что $\kappa<0$.

Рассмотрим автомодельные решения системы (1.1) вида $u_{\alpha}=u_{\alpha}(x / t)-$ волны Римана и ударные волны.

1.1. Волны Римана. Гиперболическая система (1.1) имеет два семейства характеристик - медленные и быстрые со скоростями $c_{1}$ и $c_{2}$, соответственно, $c_{1} \leqslant c_{2}$. В работе [4] изучены решения системы (1.1) вида $u_{\alpha}=u_{\alpha}(\theta(x, t))$, где $\theta$ - произвольная функция $x$ и $t$, и найдены выражения для характеристических скоростей. Если $\theta=x / t$, то эти решения называются волнами Римана. На рис. 1.1 на плоскости $\left(u_{1}, u_{2}\right)$ представлены два ортогональных семейства интегральных кривых, соответствующих изменениям величин $u_{1}$ и $u_{2}$ в быстрых и медленных волнах Римана. В рассматриваемом случае $\kappa<0$ быстрым волнам соответствуют штриховые линии, а медленным - сплошные. Стрелки на рис. 1.1 указывают направления убывания скоростей $c_{\alpha}$ вдоль интегральных кривых. В автомодельных волнах Римана изменению величин $u_{\alpha}$ с ростом $t$ соответствует движение вдоль интегральной кривой в направлении убывания характеристической скорости. 


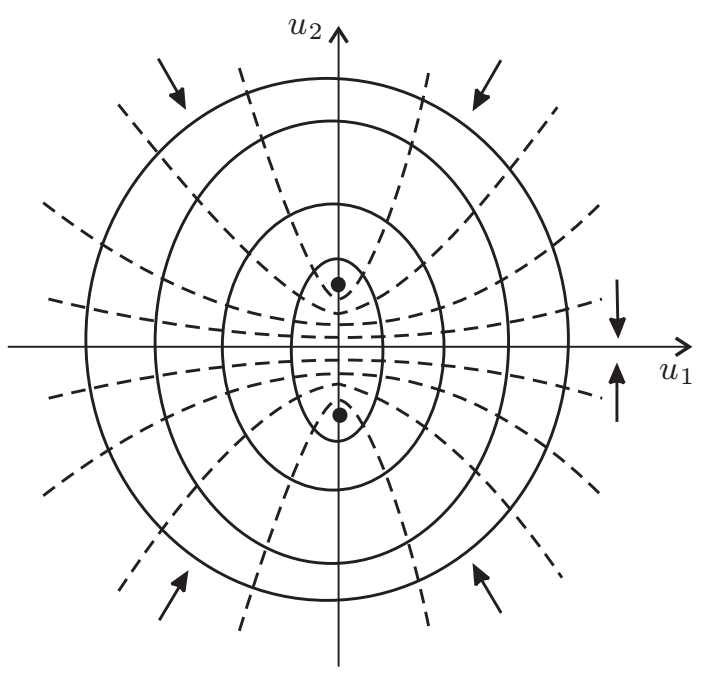

Рис. 1.1. Интегральные кривые простых волн $(\kappa<0)$.

1.2. Ударные волны. Системе уравнений (1.1) соответствуют следующие соотношения на разрыве [4], которые выражают законы сохранения поперечных компонент импульса и должны выполняться при отсутствии внешних воздействий:

$$
\left[\frac{\partial R}{\partial u_{\alpha}}\right]-W\left[u_{\alpha}\right]=0, \quad \alpha=1,2,
$$

где $W=d x / d t$ - скорость разрыва. Квадратными скобками обозначены скачки величин на фронте разрыва: $[v]=v^{1}-v^{\mathrm{r}}$. Здесь и далее верхние индексы $l$ и $r$ характеризуют значение величины за и перед разрывом, соответственно.

Исключив $W$, можно записать уравнение ударной адиабаты:

$$
\left(u_{1}^{2}+u_{2}^{2}-U_{1}^{2}-U_{2}^{2}\right)\left(U_{1} u_{2}-U_{2} u_{1}\right)+\frac{2 g}{\kappa}\left(u_{1}-U_{1}\right)\left(u_{2}-U_{2}\right)=0,
$$

где $U_{1}$ и $U_{2}-$ значения величин $u_{1}$ и $u_{2}$ перед разрывом. Величины за разрывом удовлетворяют уравнению ударной адиабаты и обозначаются по-прежнему $u_{1}$ и $u_{2}$. На рис. 1.2 на плоскости $\left(u_{1}, u_{2}\right)$ изображена ударная адиабата (точка $A$ с координатами $\left(U_{1}, U_{2}\right)$ отвечает состоянию перед разрывом).

Не все состояния $u_{1}, u_{2}$, принадлежащие ударной адиабате, могут быть состояниями за фронтом скачка. Если соотношения на разрывах представлены системой (1.2), то разрывные решения должны удовлетворять требованиям эволюционности [4]

$$
\begin{aligned}
& c_{2}^{\mathrm{r}} \leqslant W, \quad c_{1}^{\mathrm{l}} \leqslant W \leqslant c_{2}^{\mathrm{l}}, \\
& c_{1}^{\mathrm{r}} \leqslant W \leqslant c_{2}^{\mathrm{r}}, \quad W \leqslant c_{1}^{\mathrm{l}} .
\end{aligned}
$$

Соотношения (1.3) и (1.4) определяют соответственно эволюционные быстрые и медленные ударные волны. Для наглядного представления неравенств (1.3) и (1.4) 


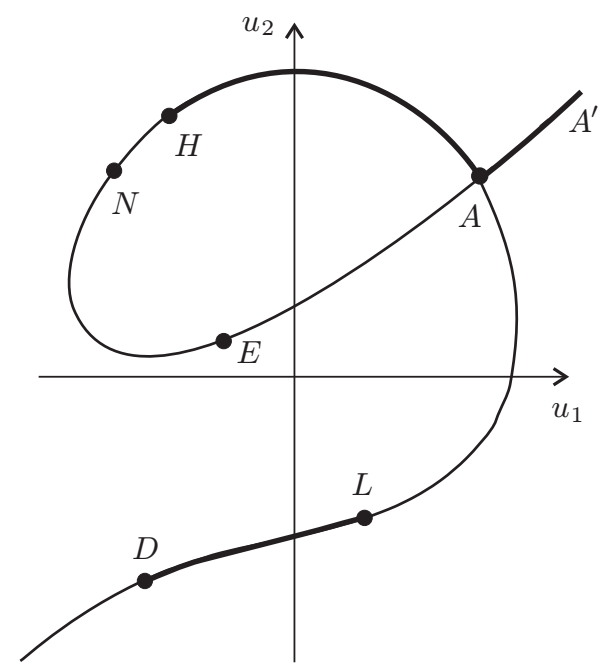

Рис. 1.2. Ударная адиабата $(\kappa<0)$.

удобно пользоваться диаграммой эволюционности (рис. 1.3), где на взаимно ортогональных осях отложены скорости $c_{1,2}$, участвующие в неравенствах, и выделены области на плоскости, где выполняется каждое из неравенств [4]. При фиксированном состоянии перед фронтом вдоль горизонтальной оси диаграммы эволюционности скорость разрыва, а также скорости характеристик воспроизводятся в одном и том же масштабе. Вдоль вертикальной оси сохранены только неравенства между $W, c_{1}^{1}$ и $c_{2}^{1}$. Области, где выполняются оба неравенства каждой из систем $(1.3),(1.4)$, образуют на плоскости заштрихованные прямоугольники.

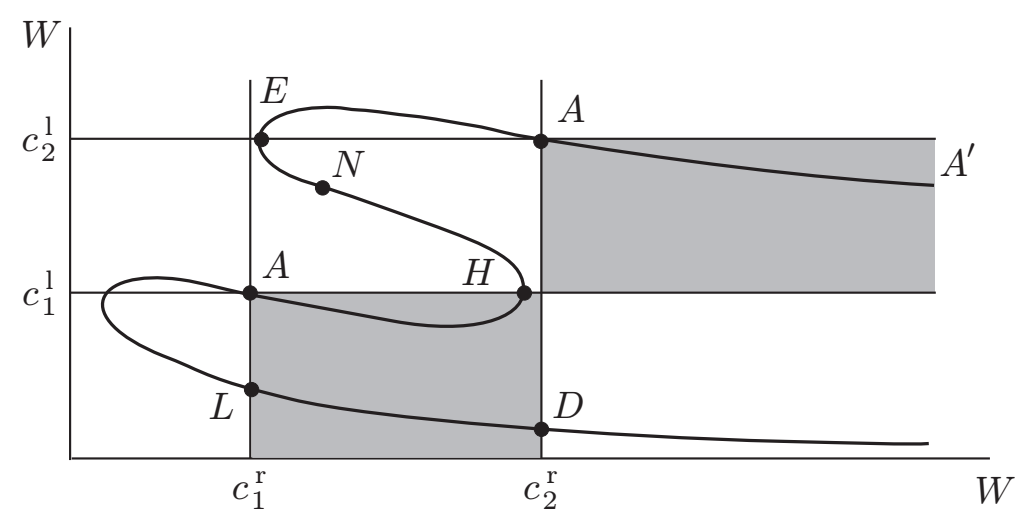

Рис. 1.3. Диаграмма эволюционности $(\kappa<0)$.

Соотношения на разрыве (1.2) позволяют найти скорость $W$ и другие величины вдоль ударной адиабаты и отобразить ударную адиабату на указанную плоскость. При этом значения $W$ правильно представляются по горизонтальной оси и лишь 
качественно по вертикальной. На рис. 1.3 начальная точка $A$ представлена двумя точками в силу ее положения на двух пересекающихся ветвях ударной адиабаты. Отрезки ударной адиабаты, которые попали в эволюционные прямоугольники, соответствуют эволюционным быстрым ударным волнам в верхнем эволюционном прямоугольнике и медленным - в нижнем. Те же отрезки на ударной адиабате на плоскости $\left(u_{1}, u_{2}\right)$ на рис. 1.2 показаны жирными сплошными отрезками. На рис. 1.2 и 1.3 одинаковые буквы относятся к одним и тем же точкам.

На рис. 1.2 и 1.3 отмеченные точки $H, L, D, E$ называются точками Жуге: в этих точках скорость ударной волны совпадает с одной из характеристических скоростей перед или за скачком. Точки Жуге являются граничными точками эволюционных

интервалов. Ударные волны, отвечающие точкам Жуге, играют важную роль в построении автомодельных решений. $\mathrm{K}$ ним в автомодельных решениях спереди или сзади могут непосредственно примыкать волны Римана того же типа, что и ударные волны.

Выделенные эволюционные отрезки $A H$ и $L D$ отвечают возможным состояниям за медленными эволюционными ударными волнами, луч $A A^{\prime}$ - возможным состояниям за быстрыми эволюционными ударными волнами.

На рис. 1.2 и 1.3 отмечена характерная точка $N$, симметричная точке $A$ относительно оси $u_{2}$ (рис. 1.2). В соответствующем скачке $A \rightarrow N$ величина $u_{1}$ меняет знак, а величина $u_{2}$ неизменна.

В рассматриваемом случае все эволюционные разрывы удовлетворяют требованию неубывания энтропии [4].

Для того чтобы сформулировать более строгое правило отбора реализующихся участков ударной адиабаты, необходимо провести исследование структуры ударных волн. Постановка этой задачи требует уточнения модели среды и выходит за рамки классической теории упругости. Этот вопрос рассмотрен в следующем разделе.

\section{2. НЕЛИНЕЙНЫЕ КВАЗИПОПЕРЕЧНЫЕ ВОЛНЫ В КОМПОЗИТНЫХ СРЕДАХ}

Рассмотрим систему уравнений, описывающую поведение нелинейных волн в упругих композитных материалах, т.е. в упругой среде, обладающей внутренней структурой, связанной с периодичностью свойств среды:

$$
\begin{aligned}
& \frac{\partial u_{1}}{\partial t}+\frac{\partial}{\partial x}\left(\frac{\partial R\left(u_{1}, u_{2}\right)}{\partial u_{1}}\right)=\mu \frac{\partial^{2} u_{1}}{\partial x^{2}}+m \frac{\partial^{2} u_{2}}{\partial x^{2}}, \\
& \frac{\partial u_{2}}{\partial t}+\frac{\partial}{\partial x}\left(\frac{\partial R\left(u_{1}, u_{2}\right)}{\partial u_{2}}\right)=-m \frac{\partial^{2} u_{1}}{\partial x^{2}}+\mu \frac{\partial^{2} u_{2}}{\partial x^{2}},
\end{aligned}
$$

Эта система отличается от системы (1.1) тем, что учитывает диссипативные и дисперсионные эффекты, которые существенны при описании структуры разрывов. Члены, описывающие дисперсию, содержат множитель $m$. Члены, содержащие $\mu$, обуславливают диссипацию. Предположим, что при малой нелинейности члены, описывающие дисперсию и диссипацию, сохраняют тот же вид, что и в линейных волнах. Вид дисперсионных членов в (2.1) соответствует одному из вариантов, полученных в [6]. 
Члены, содержащие $m$ и $\mu$, имеют более высокий порядок дифференцирования, чем члены в левой части уравнений (2.1), поэтому при изучении крупномасштабных явлений система (2.1) будет совпадать с (1.1). Будем полагать, что в (2.1) вид функции $R\left(u_{1}, u_{2}\right)$ тот же, что и в (1.1).

2.1. Структура разрывов. Будем искать решения системы $(2.1)$ вида $u_{\alpha}=$ $u_{\alpha}(\xi)$, где $\xi=-x+W t, \quad W=$ const - скорость разрыва, удовлетворяющие следующим условиям: $u_{\alpha}(\xi) \rightarrow U_{\alpha}$ при $\xi \rightarrow-\infty \quad\left(U_{\alpha}-\right.$ значения, соответствующие состоянию перед разрывом $), u_{\alpha}(\xi) \rightarrow u_{\alpha}$ при $\xi \rightarrow+\infty \quad\left(u_{\alpha}-\right.$ значения, соответствующие состоянию за разрывом, т.е. состоянию, принадлежащему некоторой точке ударной адиабаты). При $\mu \rightarrow 0$ и $m=0$ эти решения переходят в разрывы, изменение величин в которых совпадает с изменением величин в соответствующем квазипоперечном разрыве.

Для функций $u_{\alpha}(\xi)$ имеем систему двух обыкновенных дифференциальных уравнений второго порядка. Один раз проинтегрировав по $\xi$, получим систему уравнений

$$
\begin{array}{r}
\mu \frac{d u_{1}}{d \xi}+m \frac{d u_{2}}{d \xi}=-\frac{\partial Z}{\partial u_{1}}, \\
-m \frac{d u_{1}}{d \xi}+\mu \frac{d u_{2}}{d \xi}=-\frac{\partial Z}{\partial u_{2}}
\end{array}
$$

где

$$
\begin{aligned}
Z\left(u_{1}, u_{2}\right) & =-R\left(u_{1}, u_{2}\right)+\frac{1}{2} W\left(u_{1}^{2}+u_{2}^{2}\right)+Q_{1} u_{1}+Q_{2} u_{2}, \\
Q_{\alpha} & =U_{\alpha}\left(f-W+(-1)^{\alpha} g-\kappa\left(U_{1}^{2}+U_{2}^{2}\right)\right) .
\end{aligned}
$$

Заметим, что

$$
\mu\left[\left(\frac{d u_{1}}{d \xi}\right)^{2}+\left(\frac{d u_{2}}{d \xi}\right)^{2}\right]=-\frac{d Z}{d \xi}>0 .
$$

Система (2.2) с точностью до обозначений совпадает с системой, описывающей нелинейные электромагнитные волны в слабоанизотропных магнетиках [3], [5].

В [1], [3] при изучении нелинейных волновых процессов в случае, когда при описании структуры разрывов существенны процессы дисперсии и диссипации, было показано, что множество допустимых значений за разрывом $u_{\alpha}$ существенно зависит от величины отношения $m / \mu$.

Координаты особых точек системы (2.2) определяются системой уравнений

$$
\frac{\partial Z}{\partial u_{1}}=0, \quad \frac{\partial Z}{\partial u_{2}}=0 .
$$

Подчеркнем, что в случае $m / \mu \gg 1$ интегральные кривые системы $(2.2)$ близки к линиям уровня $Z\left(u_{1}, u_{2}\right)=$ const. На рис. 2.1 представлены эти линии уровня при следующих значениях параметров: $f=1, g=1, \kappa=-2, \quad W=4.6$ и $U_{1}=1$, $U_{2}=0.1$ (точка $A$ ).

Наибольший интерес представляет интервал $W_{E}<W<W_{H} \quad\left(W_{E}\right.$ и $W_{H}-$ скорости разрывов $A \rightarrow E$ и $A \rightarrow H$, соответственно, см. рис. 1.2, 1.3), в котором 


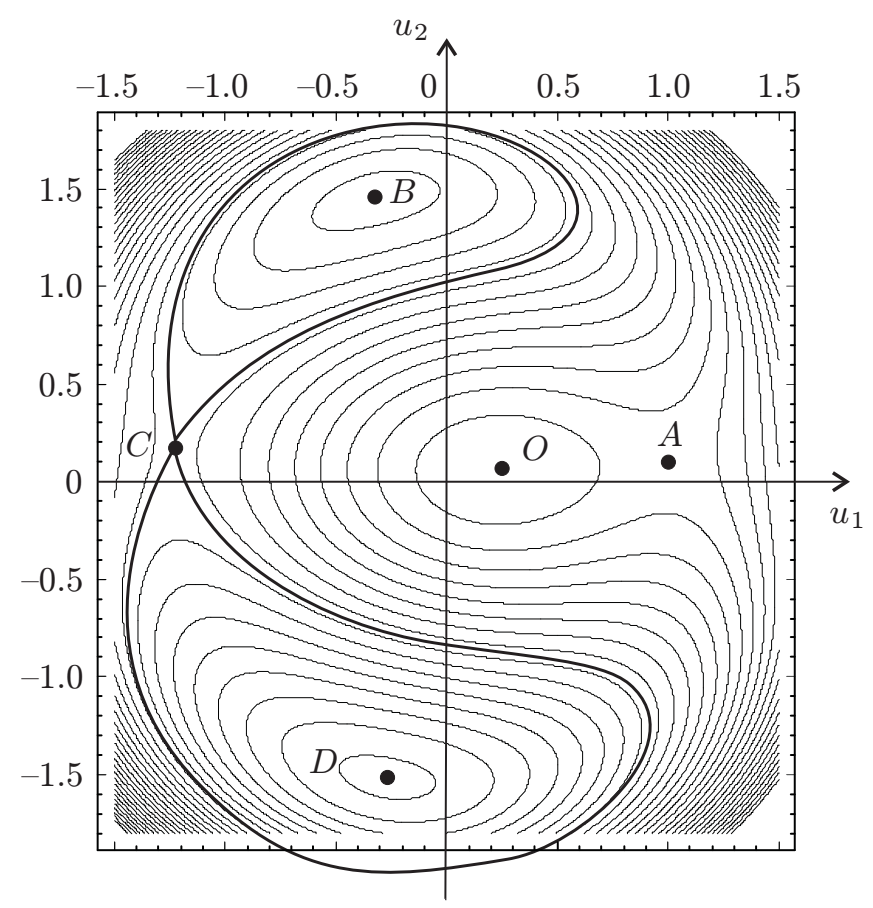

Рис. 2.1. Линии уровня.

у системы (2.2) существует пять особых точек: седло $A$ (начальная точка ), два устойчивых фокуса $B$ и $D$ (минимумы функции $\left.Z\left(u_{1}, u_{2}\right)\right)$, седло $C$ и неустойчивый фокус $O$ (максимум функции $\left.Z\left(u_{1}, u_{2}\right)\right)$.

Каждая из двух интегральных кривых, вышедших из точки $A$, при $\xi \rightarrow+\infty$ должна прийти в одну из особых точек $B, D, C$. Приход интегральной кривой в седловую точку $C$ следует рассматривать как исключительный случай, который возможен при специальном дискретном наборе значений скорости $W=W_{i}^{*}$.

Число оборотов, которые сделает интегральная кривая вокруг выделенной на рис. 2.1 в виде жирной "восьмерки" линии уровня, прежде чем остановится в стационарной точке $C$, зависит от угла наклона интегральной кривой по отношению к линии уровня. Этот угол согласно предыдущим рассуждениям имеет порядок $\mu / m$. Если разность $Z(A)-Z(C)$ конечна, а величина $\mu / m$ мала, то число оборотов велико. Достаточно малого (порядка $\mu / m$ ) изменения $W$, чтобы число оборотов вокруг "восьмерки" изменилось на единицу. Значения $W^{*}$, при которых интегральные кривые соединяют точки $A$ и $C$, разделены малыми интервалами порядка $\mu / m$. Каждое значение $W^{*}$ соответствует точке на ударной адиабате. Эта точка лежит на априорно неэволюционной части ударной адиабаты, но поскольку ей соответствует выделенное значение $W^{*}$, которое следует рассматривать как дополнительное соотношение на разрыве, то разрыв $A \rightarrow C$ эволюционный. Разрывы такого типа будем называть особыми. 
На рис. 2.2, 2.3 приведены примеры стационарных структур особых разрывов, полученные численно для значений параметров диссипации $\mu=0.05$, дисперсии $m=1.5(m / \mu=30)$ и прежних значений $U_{1}=1, \quad U_{2}=0.1, \quad f=1, \quad g=1$, $\kappa=-2$. На рис. 2.2 представлены простые структуры особых разрывов, на рис. $2.3-$ сложные структуры для больших значений $W=W^{*}$, демонстрирующие различные виды обхода выделенной на рис. 2.1 "восьмерки".

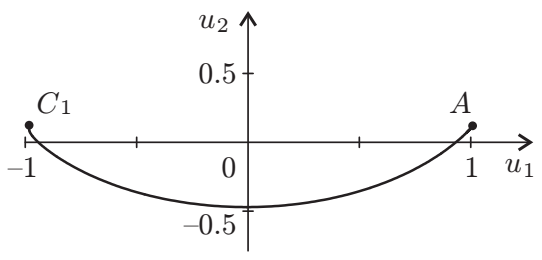

$W=4.072$

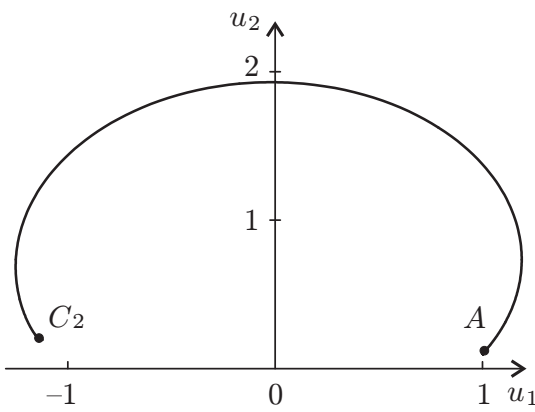

$W=4.32$

Рис. 2.2. Стационарные структуры особых разрывов.

При $m / \mu=30$ в результате численного расчета найден набор значений набор $W_{i}^{*}$, $i=1,2, \ldots, 7$, при которых одна из двух возможных интегральных кривых, вышедших из начальной седловой точки $A$ в противоположных направлениях, приходит в седловую точку $C_{i}, \quad i=1,2, \ldots, 7$. Точки $C_{i}$ соответствуют особым разрывам (рис. 2.4). Каждое значение $W_{i}^{*}$ соответствует определенному набору стационарных точек системы (2.2), выделяющих эволюционные отрезки на ударной адиабате. На рис. 2.4 на графике ударной адиабаты и на рис. 2.5 на диаграмме эволюционности жирными линиями и черными точками отмечены возможные состояния за допустимыми разрывами при начальном состоянии $A$. Допустимыми считаются разрывы, имеющие стационарную структуру при наличии нелинейности и дисперсии. Эволюционные отрезки $A H$ и $L D$ ударной адиабаты разбиваются на участки, причем часть из них не отвечает допустимым разрывам. При увеличении значения $m / \mu$ число отдельных точек и отрезков ударной адиабаты, соответствующих допустимым разрывам, увеличивается.

В рассматриваемом случае координаты точки $A$ выбраны так, что она находится вблизи оси $u_{1}$. Изучение картины интегральных кривых при увеличении координаты $u_{2}$ точки $A$ показало, что наличие отдельных точек $C_{i}$ возможно при существенном уменьшении значения параметра $\mu / m$.

При $m=0$ все эволюционные разрывы допустимы (см. рис. 1.2, 1.3) [4].

2.2. Неединственность решений автомодельной волновой задачи. Исследование структуры разрывов и определение множества допустимых разрывов конкретизирует сформулированную в разделе 1 крупномасштабную модель. Покажем, что крупномасштабная модель не гарантирует единственности решений. Неединственность возникает вследствие присутствия на ударной адиабате множества 


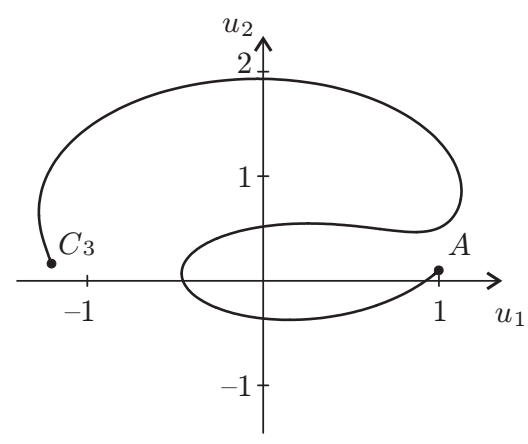

$W=4.386$

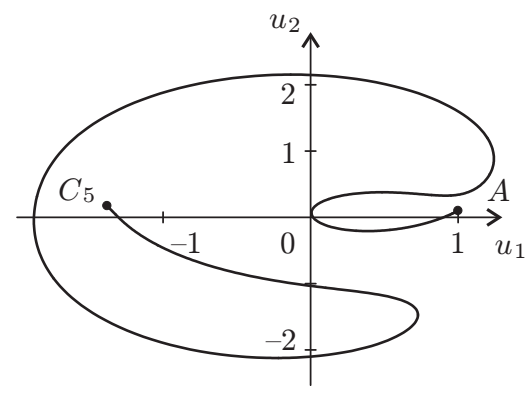

$W=5.12$

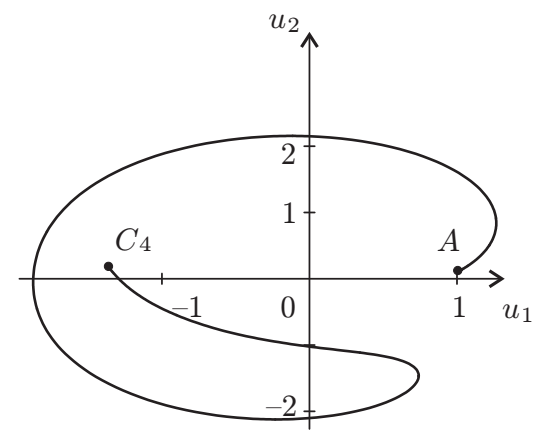

$W=5.1$

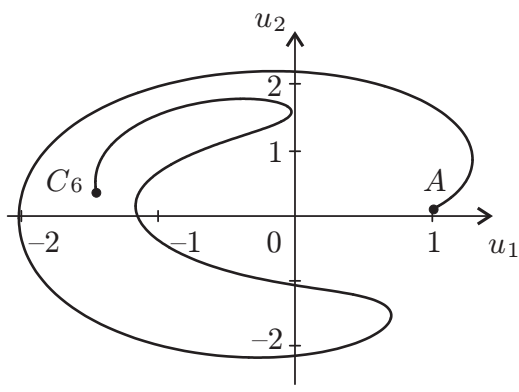

$W=5.41$

Рис. 2.3. Стационарные структуры особых разрывов.

изолированных точек с выделенными значениями скорости разрыва $W_{i}^{*}$. Эти точки лежат на диаграмме эволюционности внутри прямоугольника $c_{1}^{\mathrm{r}} \leqslant W \leqslant c_{2}^{\mathrm{r}}$, $c_{1}^{\mathrm{l}} \leqslant W \leqslant c_{2}^{\mathrm{l}}$ (рис. 2.5 ) и отвечают особым разрывам.

Рассмотрим пример автомодельной волновой задачи, в решении которой присутствуют особые разрывы. Задача о распаде произвольного разрыва для системы уравнений (2.1) формулируется следующим образом: волны распространяются в области $x>0$, начальные условия $(t=0)$ при $x>a$ соответствуют координатам точки $A$ на плоскости $\left(u_{1}, u_{2}\right)$ (рис. 2.4), при $0<x<a$ - точке $P$. Волны Римана и ударные волны умеренной амплитуды могут распространяться перед или за особым разрывом. Быстрые волны распространяются перед особым разрывом, а медленные волны - за ним.

Одно из решений сформулированной задачи представляет собой следующую последовательность волн: впереди распространяется быстрая ударная волна $A \rightarrow A_{p}$ (рис. 2.4), которая переводит состояние $A$ в такую точку $A_{p}$, что состояние за особым разрывом $A_{p} \rightarrow C_{p}$ характеризуется точкой $C_{p}$, представляющей состояние перед медленной ударной волной $C_{p} \rightarrow P$. Медленная ударная волна $C_{p} \rightarrow P$ распространяется за особым разрывом $A_{p} \rightarrow C_{p}$. Очевидно, что подобных решений имеется столько же, сколько выделенных точек $C_{i}$ на ударной адиабате, соответствующей начальной точке $A$ (в рассматриваемом случае таких точек семь). 


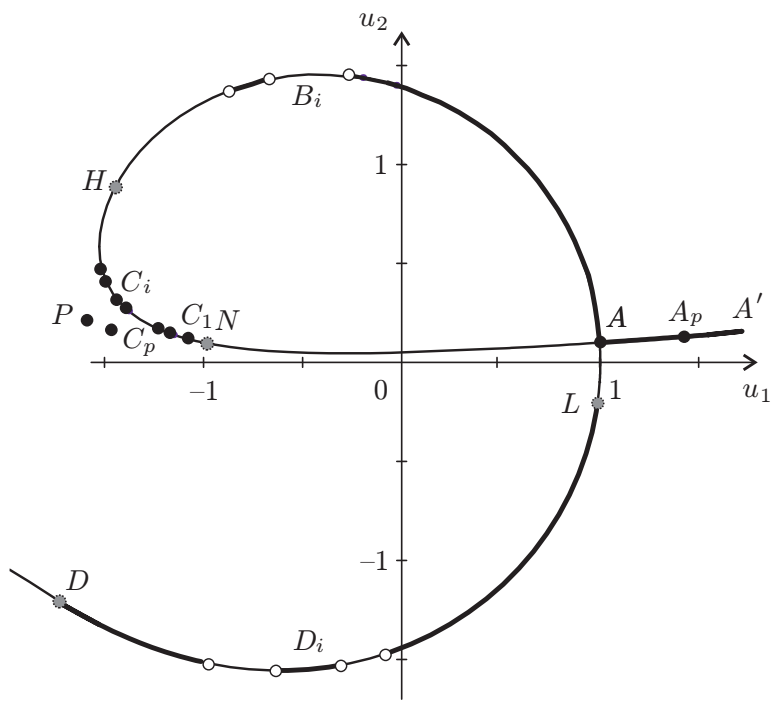

Рис. 2.4. Допустимая часть ударной адиабаты для волн в композитных материалах.

Отметим, что в отличие от эволюционных разрывов особые разрывы могут следовать один за другим. Поэтому наряду с описанными выше могут существовать другие решения, содержащие последовательности особых разрывов.

Качественный анализ решений показывает, что при заданном начальном состоянии справа от разрыва и различных состояниях слева от разрыва существует конечная область граничных значений на плоскости $\left(u_{1}, u_{2}\right)$, для которых решение автомодельной задачи не единственно. K неединственности решений задачи приводит наличие множества допустимых особых разрывов. Более детальное исследование решений и анализ проблемы отбора решений в области неединственности проводится ниже на основе численного решения обобщенной задачи о распаде произвольного разрыва для системы уравнений (2.1).

2.3. Автомодельные асимптотики в области неединственности как предел нестационарных решений системы уравнений в частных производных. Обобщенная задача о распаде произвольного разрыва формулируется следующим образом: для системы уравнений (2.1) начальные условия при $t=0$ задаются в виде

$$
u_{\alpha}= \begin{cases}u_{\alpha}^{\mathrm{l}} & \text { при } x<a, \\ u_{\alpha}^{0}(x) & \text { при } a \leqslant x \leqslant b, \quad \alpha=1,2, \quad a, b, u_{\alpha}^{\mathrm{l}}, u_{\alpha}^{\mathrm{r}}=\text { const. } \\ u_{\alpha}^{\mathrm{r}} & \text { при } b<x,\end{cases}
$$

Функции $u_{\alpha}^{0}(x)$ определяют решение сформулированной задачи о распаде произвольного разрыва, в том числе, как будет показано ниже, ту или иную асимптотику при $t \rightarrow \infty$. 


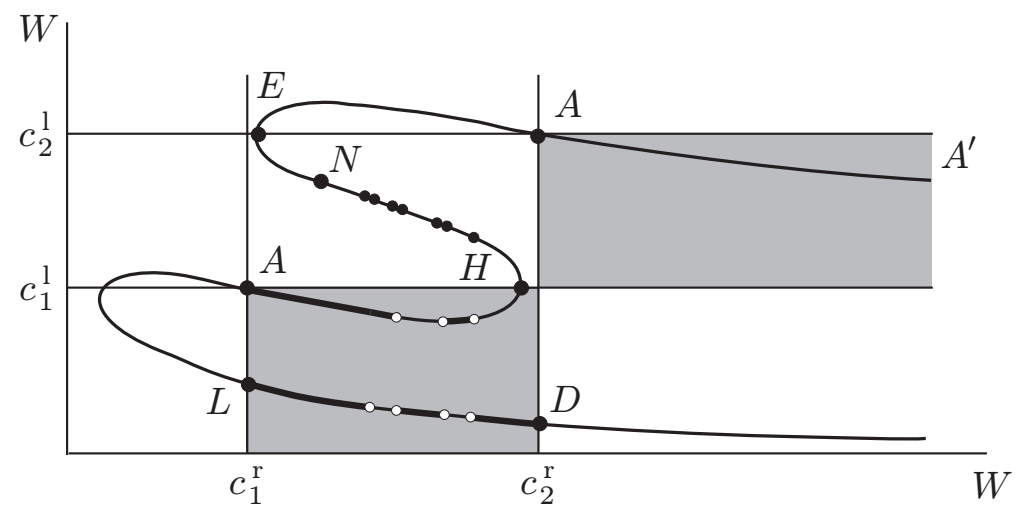

Рис. 2.5. Допустимая часть ударной адиабаты на диаграмме эволюционности.

Ниже приводятся результаты численного решения ряда начально-краевых задач для уравнений (2.1), для которых описанные в п. 2.2 автомодельные решения могут представлять асимптотики при $t \rightarrow \infty$. В ходе решения уравнения (2.1) были записаны в виде неявных нелинейных разностных уравнений, к которым сначала применялся метод Ньютона, а затем метод матричной прогонки [7]. Расчет проводился в области $t \geqslant 0,0 \leqslant x \leqslant x^{\mathrm{r}}$ с неподвижными правой и левой границами.

Волны, содержащиеся в решении, обладают разными скоростями распространения, поэтому с увеличением времени $t$ между отдельными возмущениями будут появляться отрезки (длина которых увеличивается с возрастанием $t$ ), соответствующие постоянным значениям $u_{1}, u_{2}$, а структуры ударных волн при $t \rightarrow \infty$ будут стремиться к стационарным. В силу этого для идентификации волн нужно получить решение при достаточно больших временах $t$. В связи с этим необходимо брать достаточно большой отрезок оси $x$, чтобы влияние правой границы не исказило решение. Влияние на решение левой границы устраняется соответствующим выбором параметра $f$, который в данном случае выбран так, чтобы возмущения распространялись направо.

Численно проведено исследование устойчивости стационарных структур и, в частности, структур особых разрывов по отношению к различным одномерным возмущениям. С этой целью для системы (2.1) были выбраны следующие начально-краевые условия: правые граничные условия $u_{\alpha}^{\mathrm{r}}$ (при $x=x^{\mathrm{r}}, t \geqslant 0$ ) соответствуют координатам начальной точки $A$, левые граничные условия $u_{\alpha}^{l}$ (при $x=0, t \geqslant 0$ ) соответствуют координатам точки $C_{i}$, в качестве начального условия задавались различные возмущения функций $u_{\alpha}^{0}(x)$, причем функции $u_{\alpha}^{0}(x)$ представляли собой решение системы $(2.2)$ для данных значений $u_{\alpha}^{\mathrm{r}}$ и скорости $W^{*}$, соответствующей выбранной точке $C_{i}$. Возмущения $\Delta u_{\alpha}(x)$ задавались перед исследуемой стационарной структурой. В зависимости от амплитуды и ширины возмущения решение в виде стационарной структуры либо восстанавливалось с ростом времени, либо распадалось на систему волн. Численные эксперименты показали, что стационарные структуры особых разрывов устойчивы по отношению к возмущениям не только малой, но и конечной амплитуды. В случае, когда возмущения были так велики, 
что происходил распад исходной волны, с течением времени формировалось решение, состоящее из последовательности волн: быстрая простая или ударная волна $A \rightarrow A_{p}$, особый разрыв $A_{p} \rightarrow C_{1}$ (точка $C_{1}-$ расположена рядом с точкой $N$, см. рис. 2.4) и движущаяся с меньшей скоростью медленная простая или ударная волна. Волна $A \rightarrow C_{1}$ во всех проведенных численных экспериментах восстанавливалась после взаимодействия с любыми возмущениями.

Ниже описаны результаты численного решения начально-краевой обобщенной задачи о распаде произвольного разрыва при различных начальных данных, т.е. при различных значениях $u_{\alpha}^{1}$ и $u_{\alpha}^{0}(x)$. Параметры задачи были выбраны следующим образом: $m=1.5, \quad \mu=0.05, \quad f=1, \quad g=1, \quad \kappa=-2, \quad u_{1}^{\mathrm{r}}=1, \quad u_{2}^{\mathrm{r}}=0.1$ (точка $A$ на рис. 2.6-2.8).

ПримеР 1. Пусть $u_{1}^{1}=-1.2, u_{2}^{1}=0.4$ (точка $P$ на рис. 2.6 ), функции $u_{\alpha}^{0}(x)$ представляют собой монотонный переход от значений $u_{\alpha}^{1}$ к значениям $u_{\alpha}^{\mathrm{r}}$ (см. pис. $\left.2.6 \mathrm{a}\right)$. На рис. $2.6 б$ представлено решение при значениях времени $T \sim 2$ (достаточно больших, чтобы волны успели разойтись) на плоскости $u_{1}, u_{2}$, на рис. $2.6 \mathrm{~B}-$ изменение функций $u_{1}$ и $u_{2}$ в зависимости от координаты $x$ в фиксированный момент времени $t$. Решение представляет последовательность волн: быстрая ударная волна $A \rightarrow A_{p}$, особый разрыв $A_{p} \rightarrow C_{1}$, медленная ударная волна $C_{1} \rightarrow P$. Особый разрыв имеет простую структуру, изображенную на рис. $2.2 \mathrm{a}$.

ПримеР 2. Пусть $u_{1}^{1}=-1.4, \quad u_{2}^{1}=1.4$ (точка $P$ на рис. 2.7 ), функции $u_{\alpha}^{0}(x)$ представляют монотонный переход от значений $u_{\alpha}^{\mathrm{l}}$ к значениям $u_{\alpha}^{\mathrm{r}}$ того же типа, что и в предыдущем примере. Решение, представленное на рис. 2.7 , состоит из последовательности волн: быстрая ударная волна $A \rightarrow A_{p}$, особый разрыв $A_{p} \rightarrow$ $C_{2}$, медленная ударная волна $C_{2} \rightarrow P$. Структура особого разрыва соответствует простой структуре, изображенной на рис. 2.26 .

ПримеР 3. Пусть $u_{1}^{1}=-1.4, u_{2}^{1}=1.4$ (точка $P$ на рис. 2.8 ), функции $u_{\alpha}^{0}(x)$ представлены на рис. 2.8а. Решение, представленное на рис. 2.8, состоит из последовательности волн: быстрая ударная волна $A \rightarrow A_{p}$, особый разрыв $A_{p} \rightarrow C_{2}$, особый разрыв $C_{2} \rightarrow C_{1}^{\prime}$ (этот особый разрыв имеет простую структуру, изображенную на рис. $2.2 \mathrm{a}$, при начальном состоянии, соответствующем точке $C_{2}$ ), медленная ударная волна $C_{1}^{\prime} \rightarrow P$.

ПримеР 4. Пусть $u_{1}^{\mathrm{l}}=-1.2, \quad u_{2}^{\mathrm{l}}=0.4$ (точка $P$ на рис. 2.9 ), функции $u_{\alpha}^{0}(x)$ представлены на рис. 2.9а. Решение состоит из последовательности волн: быстрая ударная волна $A \rightarrow A_{p}$, последовательность трех особых разрывов $A_{p} \rightarrow C_{1}$, $C_{1} \rightarrow C_{1}^{\prime}$ (этот разрыв имеет простую структуру, изображенную на рис. $2.2 \mathrm{a}$, при начальном состоянии, соответствующем точке $C_{1}$ ), $C_{1}^{\prime} \rightarrow C_{1}^{\prime \prime}$ (этот разрыв имеет простую структуру, изображенную на рис. $2.2 \mathrm{a}$, при начальном состоянии, соответствующем точке $C_{1}^{\prime}$ ) и медленная ударная волна $C_{1}^{\prime \prime} \rightarrow P$. На рис. 2.9б, 2.9в представлена последовательность трех особых разрывов с простыми структурами.

Исследование стационарных структур особых разрывов (см. п. 2.2) показало, что для различных значений параметров существуют две “простые" структуры (рис. 2.2) $A \rightarrow C_{1}$ и $A \rightarrow C_{2}$. Пусть начальные условия рассматриваемой обобщенной задачи о распаде произвольного разрыва заданы в виде “ступеньки" (примеры 1, 2). 


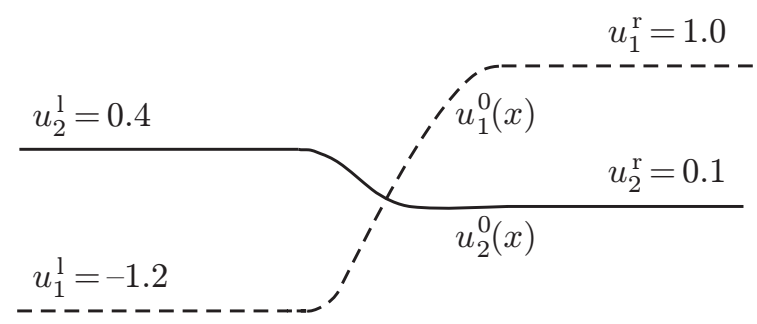

a
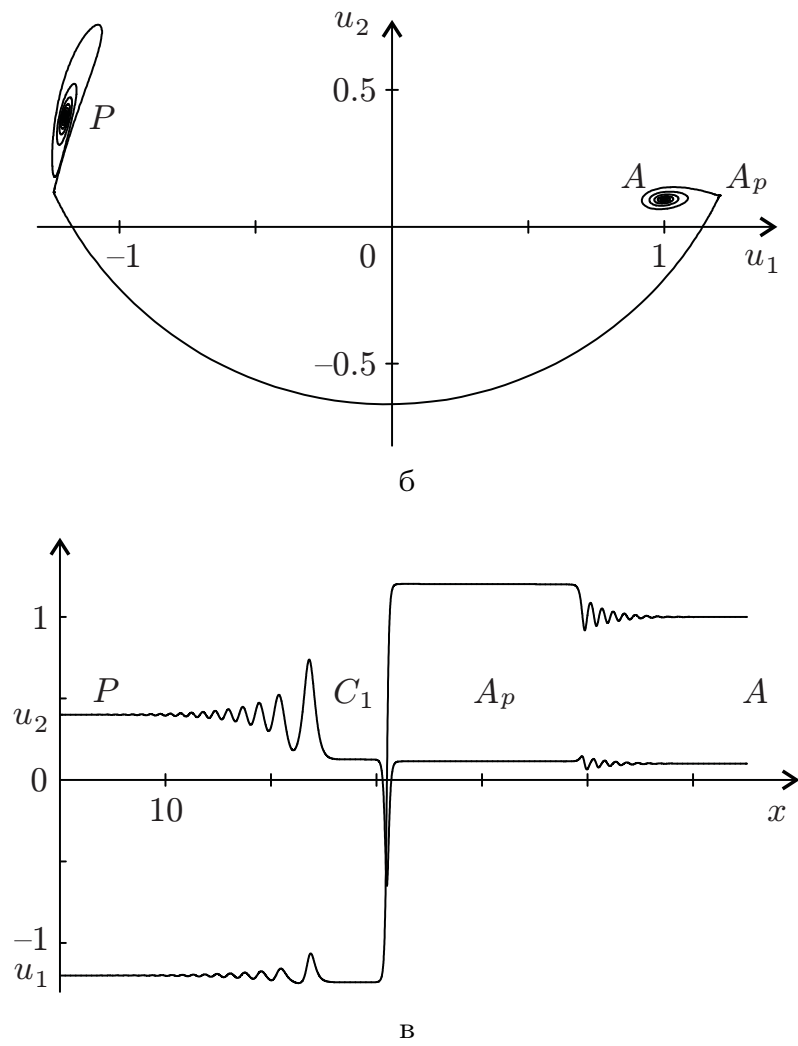

Рис. 2.6. Последовательность волн, представляющих решения для примера 1. 


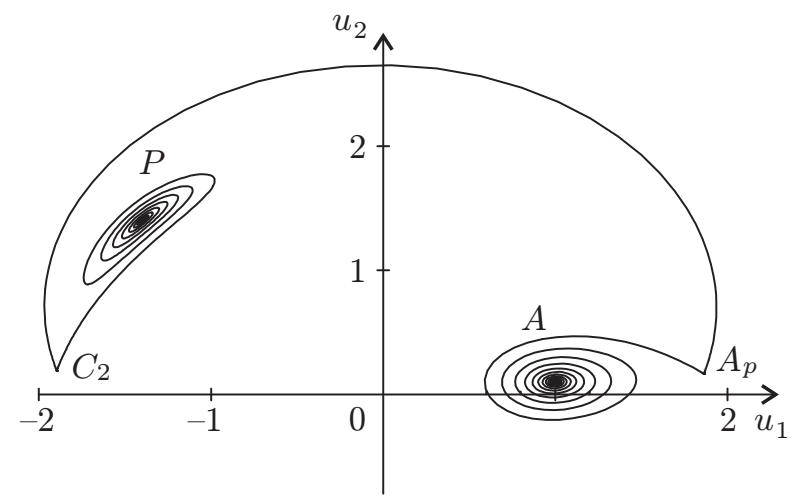

$\mathrm{a}$

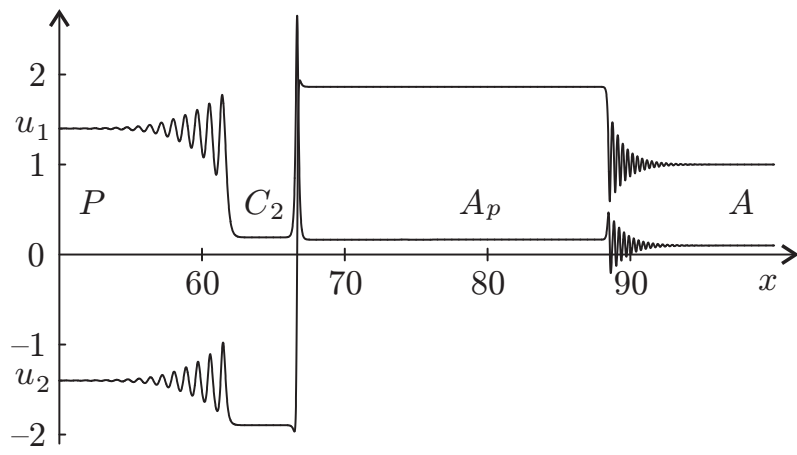

б

Рис. 2.7. То же, что на рис. 2.6, для примера 2.

Если для таких начальных данных не существует решения, не содержащего особых разрывов, то мы имеем последовательность волн, содержащих только простые структуры. Отметим, что даже в том случае, когда правое и левое граничные условия расположены в одной координатной четверти и существует решение без особых разрывов, тем не менее существует также решение, содержащее последовательность особых разрывов. Этот факт отражен в примере 3.

В примерах 1 и 4 граничные условия совпадают, а функции $u_{\alpha}^{0}(x)$ различны; соответствующие рис. 2.6 и 2.9 иллюстрируют возможность получения решений с различным количеством особых разрывов для задач с одинаковыми граничными условиями в зависимости от вида начальных функций.

Численные эксперименты, проведенные для других наборов параметров $m, \mu$, $u_{\alpha}^{\mathrm{r}}, u_{\alpha}^{\mathrm{l}}$, показали, что отмеченные в предыдущем абзаце закономерности сохраняются. Для описанной модели имеет место подобие нелинейных явлений, если сохраняются отношения величин $m / \mu$ и $x / \sqrt{\mu}$ (или $t / \sqrt{\mu}$ ). Поэтому полученные и описанные выше результаты могут рассматриваться как решения тех же уравнений с другим набором параметров. 


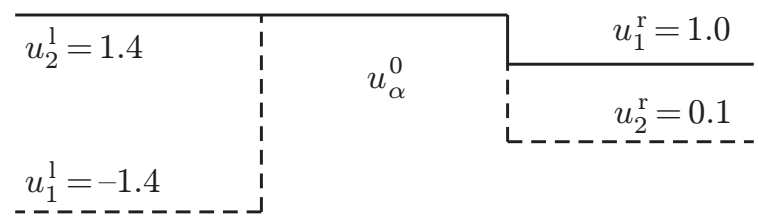

a
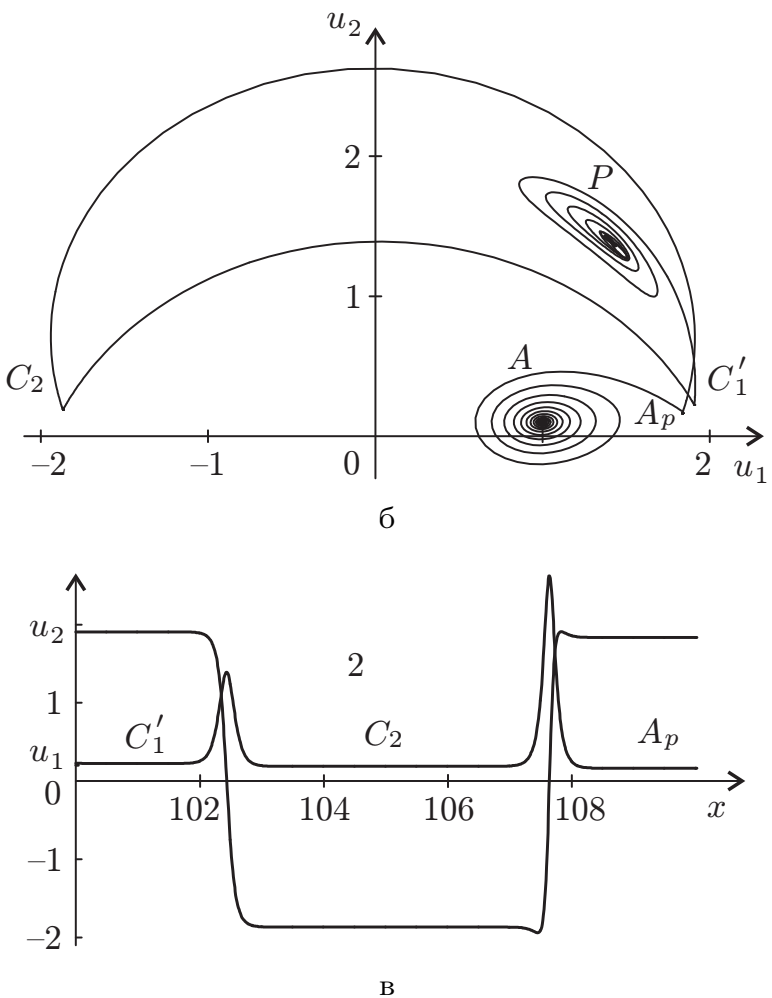

Рис. 2.8. То же, что на рис. 2.6, для примера 3. 


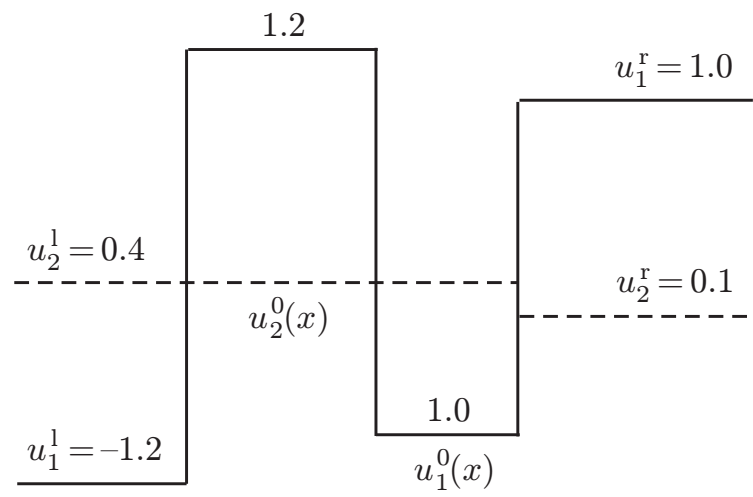

a

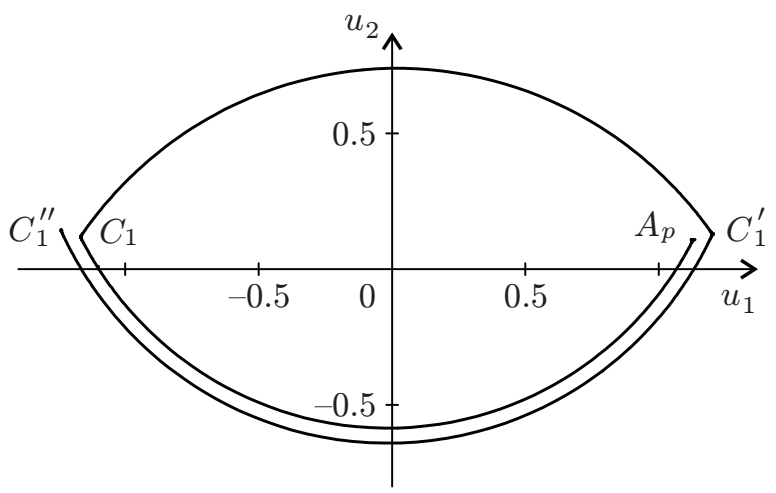

6

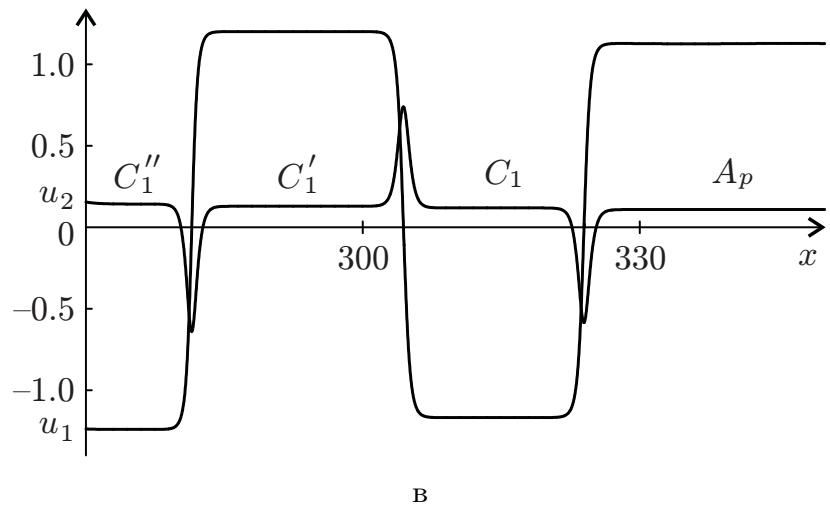

Рис. 2.9. То же, что на рис. 2.6, для примера 4. 


\section{3. ЗАКЛЮЧЕНИЕ}

Проблема неединственности решений таких стандартных автомодельных задач, описываемых нелинейными гиперболическими уравнениями, как задача о распаде произвольного разрыва, изучена в рамках более общей модели, включающей описание мелкомасштабных процессов дисперсии и диссипации внутри структуры разрывов. На основе численных экспериментов показано, что все возможные решения могут реализоваться в области неединственности как асимптотики полной системы уравнений, учитывающей мелкомасштабные процессы. Осуществление той или иной асимптотики зависит от мелкомасштабной "размазки" начального разрыва. Асимптотики, содержащие простые стационарные структуры, реализуются наиболее часто (при наиболее широких начально-краевых условиях). Асимптотики с разрывами, имеющими сложные стационарные структуры, имеют малые области притяжения в пространстве функций, задающих "размазку".

В работе [2] рассмотрена задача Коши с начальными данными в виде размазанной ступеньки для модельного уравнения, учитывающего нелинейность, дисперсию и диссипацию, и изучены асимптотики решения при больших временах. Там же показано, что при определенных начальных и граничных условиях существует колебательная нестационарная структура. В рассмотренной здесь модели композитных нелинейных упругих сред подобных асимптотик не обнаружено. Это объясняется тем, что в данной модели для любых граничных условий существует решение, содержащее простые структуры, что не выполняется для задачи, рассмотренной в работе [2].

Благодарности. Автор выражает благодарность А. Г. Куликовскому за полезные обсуждения и постоянное внимание к работе. Работа выполнена при поддержке РФФИ (грант № 05-01-00219) и программы “Математические методы нелинейной динамики".

\section{Список литературы}

[1] А. Г. Куликовский, ДАН СССР, 275:6 (1984), 1349-1352.

[2] А. Г. Куликовский, А. П. Чугайнова, ЖЖМиМФ, 44:6 (2004), 1119-1126.

[3] А. Г. Куликовский, Н. И. Гвоздовская, Тр. МИАН, 223 (1998), 63-73.

[4] А. Г. Куликовский, Е. И. Свешникова, Нелинейные волны в упругих средах, Моск. лицей, М., 1998.

[5] А. Г. Куликовский, Н. В. Погорелов, А. Ю. Семенов, Математические вопросъ численного решения гиперболических систем уравнений, Физматлит, М., 2001.

[6] Н. С. Бахвалов, М. Э. Эглит, Докл. РАН, 370:1 (2000), 1-4.

[7] А. А. Самарский, Ю. П. Попов, Разностные методы решения задач газовой динамики, Наука, М., 1978. 